\title{
REVIEW
}

\section{Ultrafast lasers—reliable tools for advanced materials processing}

\author{
Koji Sugioka ${ }^{1}$ and Ya Cheng ${ }^{2}$
}

The unique characteristics of ultrafast lasers, such as picosecond and femtosecond lasers, have opened up new avenues in materials processing that employ ultrashort pulse widths and extremely high peak intensities. Thus, ultrafast lasers are currently used widely for both fundamental research and practical applications. This review describes the characteristics of ultrafast laser processing and the recent advancements and applications of both surface and volume processing. Surface processing includes micromachining, microand nanostructuring, and nanoablation, while volume processing includes two-photon polymerization and three-dimensional (3D) processing within transparent materials. Commercial and industrial applications of ultrafast laser processing are also introduced, and a summary of the technology with future outlooks are also given.

Light: Science \& Applications (2014) 3, e149; doi:10.1038/lsa.2014.30; published online 11 April 2014

Keywords: 3D fabrication; industrial application; micromachining; nanofabrication; ultrafast laser

\section{INTRODUCTION}

Materials processing using ultrafast lasers, lasers that emit light pulses shorter than a few tens of picoseconds, was first reported in 1987 by Srinivasan et al. ${ }^{1}$, Küper and Stuke. ${ }^{2}$ They demonstrated the clean ablation of polymethyl methacrylate almost without the formation of a heat-affected zone (HAZ) using femtosecond ultraviolet excimer lasers. The ablation threshold was significantly lower than that for nanosecond laser ablation. These experiments had a significant impact and the research in this field was rapidly expanded in the 1990s. In addition, development of the chirped-pulse amplification technique in Ti:sapphire regenerative amplifiers, ${ }^{3}$ which emit energetic femtosecond pulses without inducing damage or undesirable nonlinear effects in the amplification medium, further accelerated fundamental research on ultrafast laser processing. One important feature of ultrafast laser processing is that it reduces heat diffusion to surrounding regions of the processed area. ${ }^{4}$ This feature is well adapted to the high-quality microfabrication of soft materials such as biological tissues ${ }^{5}$ and hard or brittle materials such as semiconductors and insulators ${ }^{6}$ without HAZ formation. Furthermore, suppression of heat diffusion to the surroundings improves the spatial resolution for nanoscale processing. ${ }^{7}$ Another important aspect of ultrafast laser processing is that nonlinear absorption (i.e., multiphoton absorption) can induce strong absorption even in materials that are transparent to the ultrafast laser beam. ${ }^{8,9}$ Multiphoton absorption permits not only surface processing, ${ }^{10}$ but also three-dimensional (3D) internal microfabrication of transparent materials such as glass and polymers. ${ }^{11-14}$ Davis et al. ${ }^{11}$ and Glezer et al. ${ }^{12}$ pioneered this field and demonstrated respectively optical waveguide writing and formation of nanovoid arrays inside glass in 1996. Currently, internal microfabrication is widely applied to the fabrication of photonic devices and biochips. ${ }^{15,16}$ It was also reported in 2001 that multiphoton absorption improves spatial resolution to exceed the diffraction limit, due to the nonlinearity combined with the threshold effect. ${ }^{17}$ Careful control of the laser power and scanning speed enabled a minimum fabrication resolution of $c a 18 \mathrm{~nm}$ to be achieved. ${ }^{18}$ One of the major application fields of this feature is two-photon polymerization (TPP) for the fabrication of photonic crystals, ${ }^{19}$ micromachines ${ }^{20}$ and biochips. ${ }^{21}$ In the 2000 s, it was determined that ultrafast laser irradiation at intensities near the ablation threshold forms nanoripple structures on various materials with periodicities much shorter than the wavelength. ${ }^{2-25}$ Regular arrays of conical microstructures were also produced on Si by irradiation with an ultrafast laser beam in a halogen atmosphere (e.g., $\mathrm{SF}_{6}$ or $\mathrm{Cl}_{2}$ ). ${ }^{26,27}$ The surface structures produced exhibit unique properties of anti-reflectivity, superhydrophobicity and infrared absorption.

A robust, stable and very compact fiber chirped pulse amplifier was also developed in the $2000 \mathrm{~s},{ }^{28}$ which facilitated the application of this research. More recently, a rare earth-doped laser medium was adopted to realize a compact and high-power ultrafast laser system by diode pumping, although the pulses were much broader than pulses generated by Ti:sapphire systems. ${ }^{29,30}$ Picosecond lasers based on this system that are suitable for industrial applications have become

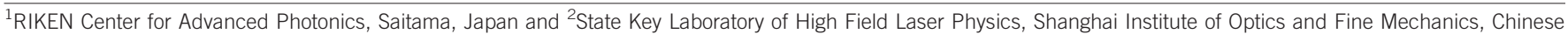
Academy of Sciences, Shanghai, China

Correspondence: Dr K Sugioka, RIKEN Center for Advanced Photonics, 2-1 Hirosawa, Wako, Saitama 351-0198, Japan

E-mail: ksugioka@riken.jp

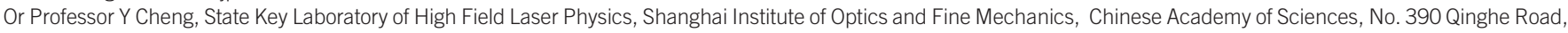

Jiading, Shanghai 201800, China

E-mail: ya.cheng@siom.ac.cn

Received 5 August 2013; revised 23 October 2013; accepted 29 October 2013 
commercially available. In the 2010s, ultrafast laser processing is thus becoming a more reliable tool for practical and industrial applications.

Here we provide a comprehensive review of ultrafast laser processing including surface micromachining, micro- and nanostructuring, nanoablation, and 3D and volume processing after discussion of the ultrafast laser processing characteristics. We then introduce commercial applications of ultrafast laser processing before a final summary and outlook are given.

\section{CHARACTERISTICS OF ULTRAFAST LASER PROCESSING}

Elimination of thermal diffusion and suppression of the HAZ Materials processing with ultrafast lasers provides unique advantages over conventional laser processing techniques that typically employ nanosecond and longer pulses. Shortening of the pulse duration to a time scale shorter than a couple of picoseconds fundamentally changes the physics behind the interaction of laser with the solid, which results in rapid and precise energy deposition into the materials. During ultrafast laser irradiation, the excitation of carriers first occurs within hundreds of femtosecond by the absorption of photons. At this stage, the lattice remains largely undisturbed. Efficient energy transfer from the electrons to the lattice occurs by electron-lattice scattering after the end of the laser pulse. ${ }^{31,32}$ The thermalization between free electrons and the lattice then typically occurs on the time scale of 1-100 ps, depending on the electron-phonon coupling strength of the material. Thermal diffusion to the surrounding area of the laser-irradiated region can thus be eliminated as heat transfer by bulk thermal conduction occurs on a time scale longer than the electron-phonon coupling time.

The suppression of thermal diffusion has important implications for the laser processing of materials. Materials processing with lasers results in phase and structural modifications induced by the interaction of light with a material. In an ideal case based on photolysis, the spatial resolution is simply determined by carrier excitation within the focal spot. However, when pulses with durations of nanosecond and longer are used, such ideal resolution cannot be achieved due to thermal diffusion. In addition, thermal effects such as melting and resolidification can also remove subtle phase and/or structural changes induced by various types of photophysical and photochemical processes. These difficulties inherent to conventional laser processing can be overcome with ultrafast laser materials processing.

\section{Deterministic optical breakdown and reproducible nanoscale resolution}

When processing transparent materials such as glass and wide-bandgap crystals with ultrafast laser pulses, the excitation of electrons from the valence band to the conduction band is initiated through nonlinear processes such as multiphoton or tunnel ionization. ${ }^{33,34}$ The electrons can then seed the subsequent electron heating or avalanche ionization, which leads to the efficient absorption of light. Although this is also possible to achieve with nanosecond lasers, in this case, the initial (seed) electrons are mainly generated by the contribution of defects and impurities, which causes the statistical character of laserinduced optical breakdown. Employing ultrafast lasers in materials processing can thus significantly reduce the fluctuation associated with the optical breakdown threshold and lead to improved reproducibility and control over the processing parameters. ${ }^{35}$

The synergetic contribution of the deterministic optical breakdown characteristics and the suppression of thermal diffusion provide a unique chance to realize deterministic nanoscale (subdiffraction-limited) fabrication with ultrafast lasers when there is an intensity threshold only above which a photoreaction occurs upon absorption. ${ }^{17,36}$ Ideally, the focal spot of the ultrafast laser beam will have a Gaussian-like spatial profile. Thus, the modified zone area can be limited to only the central area of the focal spot by adjusting the laser intensity to match the threshold intensity, as shown in Figure la. If the laser intensity is adjusted to match the straight solid line shown in Figure $1 \mathrm{~b}$ with the threshold intensity for a reaction, then the resolution for fabrication can be reduced to two-fifths of the original beam width. Thermal diffusion in ultrafast laser microprocessing is negligible; therefore, the combination of high-numerical aperture focusing and the threshold effect has enabled deep subwavelength fabrication resolutions that are far beyond the diffraction limit. ${ }^{17,18,36,37}$ In principle, there is no limit to the fabrication resolution when taking advantage of the threshold effect, because the resolution can always be improved if one keeps on reducing the difference between the chosen laser intensity and the threshold intensity. However, in practice, due to the fluctuation of the output power of the femtosecond laser, the fabrication process will become extremely unstable when the laser intensity is near the threshold intensity. Thus, a compromise has to be made between improving the resolution and maintaining the stability of the fabrication process.

\section{Internal modification and miscellaneous structural changes}

One of the most exciting features of ultrafast laser processing is the unique $3 \mathrm{D}$ capability to process within transparent materials in a space selective manner. This is enabled by the nonlinear nature of the ultrafast laser interaction with the transparent material, which confines the modification to only that within the focal volume. Combining a tight focus scheme (which is necessary to avoid self-focusing and to ensure high axial resolution) and direct writing, this approach has allowed for a wide variety of phase and structural changes inside materials, such as refractive index modification, ${ }^{11}$ the formation of nanovoids and periodic nanogratings, ${ }^{38,39}$ element redistribution, ${ }^{40}$ nanocrystallization ${ }^{41}$ and nonreciprocal writing. ${ }^{42}$ Thus, the ultrafast (particularly femtosecond) laser pulses can be used to finely tune or even completely alter the physical and chemical properties of transparent materials, which provides the possibility to induce and integrate multiple functions in a single substrate. ${ }^{16,43,44}$

\section{SURFACE PROCESSING}

\section{Micromachining, drilling and cutting}

Ultrafast lasers suppress thermal diffusion and thus reduce HAZ formation, even in high thermal conductivity materials such as metals. Figure $2 \mathrm{a}$ and $2 \mathrm{~b}$ show scanning electron microscopy (SEM) images of holes drilled in $100 \mu \mathrm{m}$ thick steel foils by ablation using laser pulses with widths of $200 \mathrm{fs}$ and $3.3 \mathrm{~ns}$, respectively. ${ }^{45}$ The femtosecond laser produces an ablated hole with a sharp edge and a steep wall with little formation of HAZ. In contrast, nanosecond laser ablation produces significant swelling around the ablated hole due to melting.

Ultrafast lasers can perform high quality micromachining of even brittle materials such as glass. Figure $3 a$ and $3 b$ show SEM images of a micromachined surface and glass cut by femtosecond laser ablation, respectively. Both images reveal that clean ablation with sharp edges was achieved without the formation of cracks. The ultrafast lasers have already been used or are being considered for use in practical applications such as substrate scribing, hole drilling, surface patterning and stent fabrication, as introduced later.

\section{Micro- and nanostructuring}

A variety of micro- and nanoscale structures are dependent on processing parameters such as the beam intensity, spatial and temporal beam 


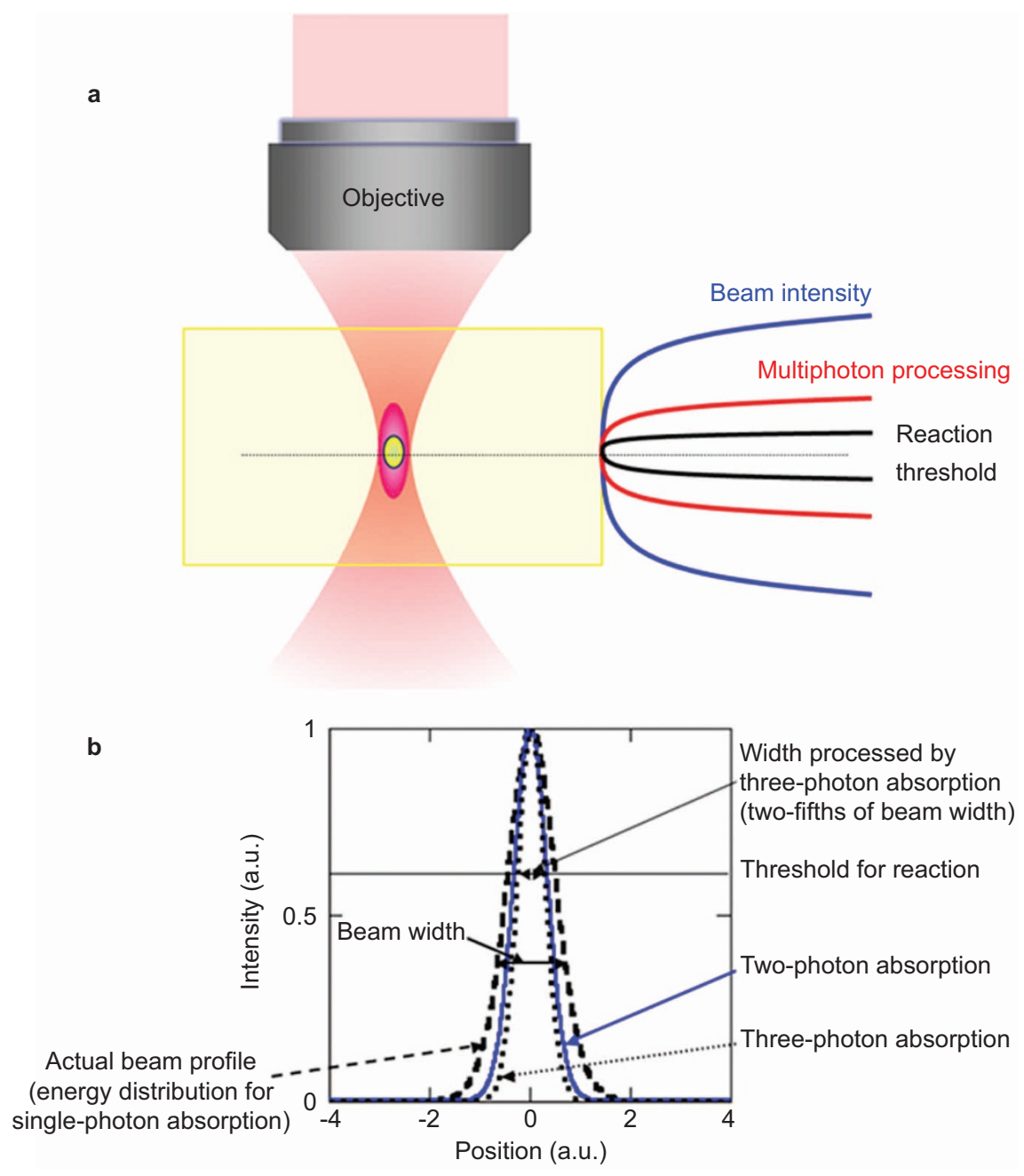

Figure 1 (a) Schematic illustration of subdiffraction-limited fabrication based on multiphoton absorption. (b) Beam profile (thick dashed line) and spatial distributions of laser energy absorbed by transparent materials by two- (solid line) and three-photon (thin dashed line) absorption. The solid horizontal line indicates the reaction threshold.

profiles, wavelength, polarization, and the processing environment (ambient gas or liquid) that can be formed on the material surface. The most well-known textured structure is nanoripples, which are formed by ultrafast laser irradiation with a fluence near the ablation threshold. Periodic grating structures, the so-called laser-induced periodic surface structures, are formed by irradiating materials with linearly polarized nanosecond or longer laser pulses. ${ }^{46}$ Such structures are understood to be formed by the interference of the incident laser light with the reflected (scattered) light. Consequently, ripples are generally oriented perpendicular to the incident polarization. The spacing of the fabricated structure is given by $\lambda / n(1 \pm \sin \theta)$, where $\lambda$ is the laser wavelength, $\theta$ is the laser incident angle and $n$ is the refractive index of the material. ${ }^{47,48}$ Therefore, the spacing is always in the order of the wavelength or greater. In contrast, periodic grating structures formed by femtosecond laser irradiation have a spacing much smaller than the laser wavelength (typically $1 / 10-1 / 5$ of $\lambda$ ). ${ }^{49}$ Nanoscale periodic structures can be formed not only on the surfaces of various materials including metals, ${ }^{50}$ ceramics $^{25,51}$ semiconductors ${ }^{52}$ and insulators, ${ }^{22,53}$ but also inside transparent materials such as glass. ${ }^{22,39}$ Figure 4 shows SEM images of nanoripples formed on Si by irradiation with linearly polarized $170 \mathrm{fs}$ laser pulses with a wavelength of $800 \mathrm{~nm}$ and an energy per pulse of 3 nJ. ${ }^{54}$ The orientation of the nanoripples formed is perpendicular to the polarization of the incident laser beam. The periodicity of the ripples is approximately $\Lambda \approx 120 \pm 10 \mathrm{~nm}$, which is smaller than one-sixth of the incident laser wavelength. Various mechanisms, including the self-organization of surface instability, ${ }^{22}$ second-harmonic generation, ${ }^{52}$ refractive index change, ${ }^{55}$ nanoplasma formation $^{56}$ and the excitation of surface plasmon polaritons, ${ }^{57}$ have been proposed for the formation of nanoripples; however, no consensus has been reached until now. Surface nanoripples produced by femtosecond laser irradiation can be applied to reduce the friction between moving components, reduce the adhesive force of microand nanocomponents and increase the adhesion of thin films and medical implants.

Another interesting and useful texture formed by ultrafast laser irradiation is regular arrays of conical microstructures, which can be produced on Si by irradiation with hundreds of femtosecond laser pulses in a halogen atmosphere (e.g., $\mathrm{SF}_{6}$ or $\mathrm{Cl}_{2}$ ), as shown in Figure $5 .^{27,58}$ The structures produced can strongly reduce incident light reflection, which is why it is named black silicon, and have significantly increased absorption even in the infrared region. ${ }^{26,59}$ This technique is effective to enhance the efficiency of photovoltaic solar 

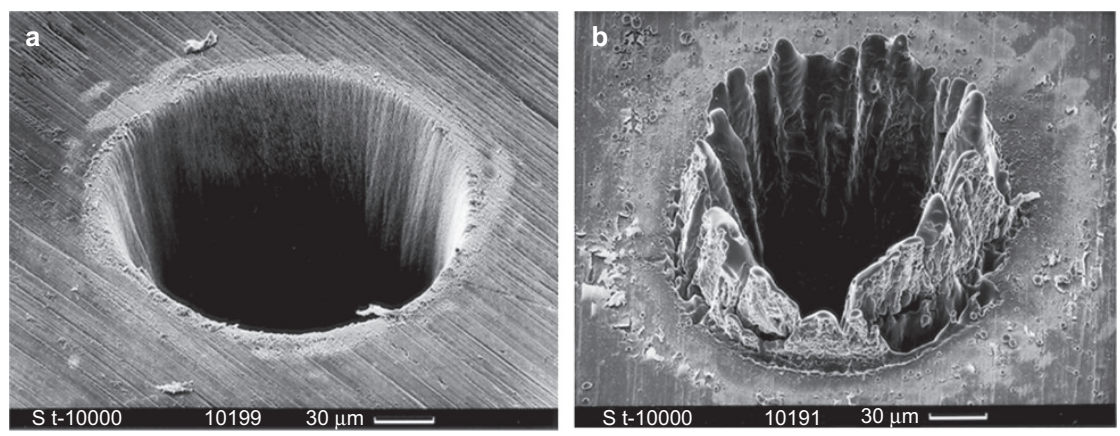

Figure 2 Holes drilled in $100 \mu \mathrm{m}$ thick steel foils by ablation using laser pulses with the following parameters: (a) pulse width: $200 \mathrm{fs}$, pulse energy: $120 \mu \mathrm{J}$, fluence: $0.5 \mathrm{~J} \mathrm{~cm}^{-2}$, wavelength: $780 \mathrm{~nm}$; and (b) pulse width: $3.3 \mathrm{~ns}$, pulse energy: $1 \mathrm{~mJ}$, fluence: $4.2 \mathrm{~J} \mathrm{~cm}^{-2}$, wavelength: $780 \mathrm{~nm}$. The scale bars represent $30 \mu \mathrm{m} .{ }^{45}$ Reproduced with permission from Springer. (0)1996 by Springer.

cells. ${ }^{60}$ Coating of such structured surfaces with a layer of silane molecules produces superhydrophobic surfaces due to the lotus effect, and these surfaces can be used to produce self-cleaning products. ${ }^{60,61}$ Similar surface structures can also be formed with other materials. ${ }^{62}$

\section{Nanoablation}

The ability of ultrafast laser processing to reduce the formation of HAZ and thereby suppress heat diffusion means that nanoablation can be performed with subwavelength resolution or less in combination with a well-defined processing threshold. In addition, the use of nonlinear multiphoton absorption can further enhance the spatial resolution compared with single-photon absorption at the same wavelength when irradiated with a Gaussian beam, because the effective absorption coefficient for $n$-photon absorption is proportional to the $n$th power of the laser intensity. ${ }^{17}$ Furthermore, the fabrication resolution can be further improved by adjusting the laser intensity to near the ablation threshold, by which only the center part of the Gaussian laser beam can exceed the ablation threshold. A combination of these effects has enabled the formation of nanohole arrays with diameters smaller than $200 \mathrm{~nm}$ on a GaN surface by femtosecond laser ablation ( $\lambda=387 \mathrm{~nm}$ and the numerical aperture of the objective lens $=0.9) .{ }^{63,64}$

In principle, the fabrication resolution can be improved as much as desired with no limitation by employing the threshold effect with precise control of the laser intensity. For example, short polymer lines with linewidths as narrow as $c a 18 \mathrm{~nm}$ have been produced using TPP with a femtosecond laser. ${ }^{18}$ However, due to the fluctuation of the femtosecond laser output power, the fabrication process becomes extremely unstable when the laser intensity is near the threshold intensity. Therefore, it is difficult to reproducibly realize superhigh resolution over a long range. This problem can be overcome by combining the threshold effect and the formation of a periodic nanograting. A nanoscale periodic grating is formed inside glass when irradiated with a linearly polarized beam, ${ }^{39}$ which means that the energy deposition inside glass is spatially modulated with nanoscale periodicity at the focal spot, as shown in Figure 6a. When the femtosecond laser intensity is intentionally reduced to a level at which only the intensity in the blue region of Figure 6a exceeds the threshold intensity, only a single cycle of the modulated energy distribution in the central area of the focal volume can be selected. It is noteworthy that in such a case, the peak laser intensity at the center of the focal spot is still much greater than the laser intensity at the edge of the blue zone (i.e., the threshold intensity); therefore, the fabrication process will be much less sensitive to the fluctuation of the laser output power than that achievable by employing only the threshold effect to achieve the same transverse resolution. Using this scheme, Figure $6 \mathrm{~b}$ shows a nanochannel with a width of $c a 40 \mathrm{~nm}$ and a length of $c a 40 \mu \mathrm{m}$ (aspect ratio: $c a$ 1000) resulting from the ablation of porous glass in water using a linearly polarized femtosecond laser beam. ${ }^{37}$

Another approach to nanofabrication is the development of novel irradiation methods that can also overcome the diffraction limit of the focused laser beam by using optical near fields. Combining a femtosecond laser beam with nanotips in scanning-probe microscopes such as scanning near-field optical microscopes, scanning tunneling microscopes and atomic force microscopes permits patterning with nanoscale resolution. ${ }^{65,66}$ Recently, nanoablation using metal and dielectric nanospheres has been extensively investigated. ${ }^{67,68}$ The mechanism for the formation of subwavelength structures involves ablation by the near-field enhanced near the nanospheres. This method can be used to fabricate nanoholes on various material surfaces including semiconductors, metals and dielectrics.
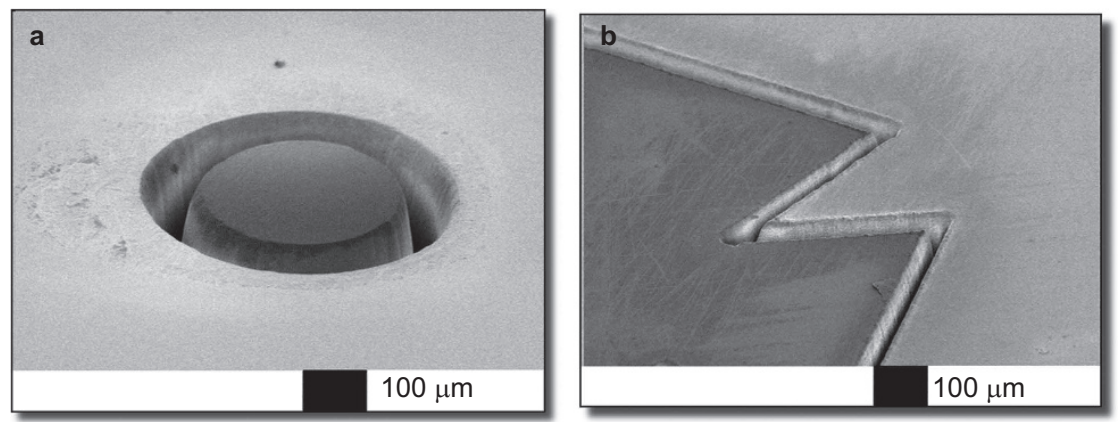

Figure 3 SEM images of (a) a micromachined surface and (b) a glass material cut by femtosecond laser ablation (Courtesy of M. Gower). SEM, scanning electron microscopy. 

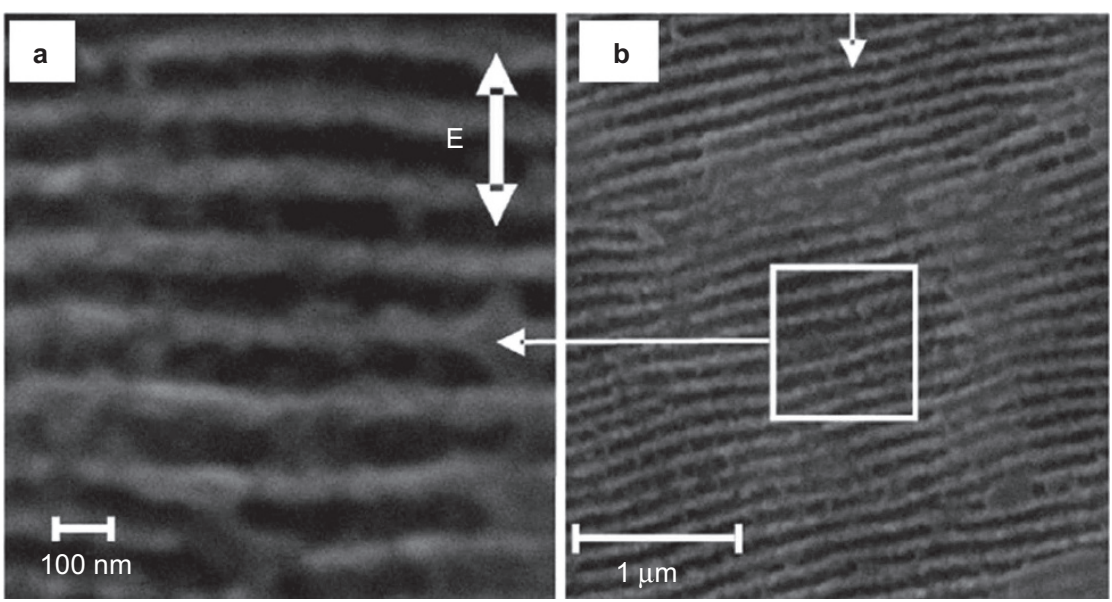

Figure 4 SEM images of nanoripples formed on Si by irradiation with linearly polarized $170 \mathrm{fs}$ laser pulses with a wavelength of $800 \mathrm{~nm}$ and an energy per pulse of $3 \mathrm{~nJ}$. (a) High-resolution image. (b) Image of wider area. ${ }^{54}$ Reproduced with permission from OSA. @2011 by the Optical Society of America. SEM, scanning electron microscopy.

\section{D AND VOLUME PROCESSING}

\section{Two-photon polymerization}

Polymers are a cost-effective material for rapid prototyping. The dominant approach to high-resolution 3D fabrication with polymer materials is TPP, of which the concept is schematically illustrated in Figure 7. The TPP technique is based on the two-photon absorption of femtosecond laser pulses in a photosensitive resin, which only occurs in the central region of the focal spot where the laser intensity exceeds the TPP threshold. ${ }^{69,70}$ Writing of predesigned $3 \mathrm{D}$ microstructures is realized by converting the liquid resin to the solid phase, point by point, using a focused femtosecond laser beam. TPP is now one of the major approaches to laser-based 3D printing with nanometer scale feature sizes. The basic concept, characteristics, range of materials and potential applications have already been reviewed. ${ }^{21,71}$ In the following, we will focus on recent advances such as improvement of the resolution, functionalization of materials and the development of novel applications.

One prominent feature of the TPP technique is the high fabrication resolution achieved by use of the threshold effect. Currently, TPP can reliably support a lateral resolution at the $100 \mathrm{~nm}$ level by the optimization of laser conditions such as the laser wavelength, pulse duration, peak intensity and repetition rate, and by exposure conditions such as numerical aperture of the objective lens and the scan speed, and finally by selection of the chemical/physical properties of the photopolymers. The axial resolution is usually significantly poorer, typically by a factor of three, due to the longitudinal intensity distribution within the focal spot. ${ }^{72,73}$ Although higher resolutions in the order of a few tens of nanometers have been demonstrated with TPP by setting the laser intensity very near the threshold intensity, the reproducibility becomes poor because of the inherent sensitivity of the fabrication process to the fluctuation of the femtosecond laser output power under these conditions. Inspired by the concept of stimulated emission depletion microscopy, Li et al. have recently overcome the limitation in TPP by developing a novel superresolution lithography scheme. The stimulated emission depletion microscopy was originally developed for the far-field nano-imaging of live cells. ${ }^{74}$ In the so-called resolution augmentation through the photo-induced deactivation lithography scheme as illustrated in Figure 8a, one laser beam (the activation beam) comprised of $800 \mathrm{~nm}$ and $200 \mathrm{fs}$ pulses was employed to induce photopolymerization in a negative-tone photoresist. ${ }^{75}$
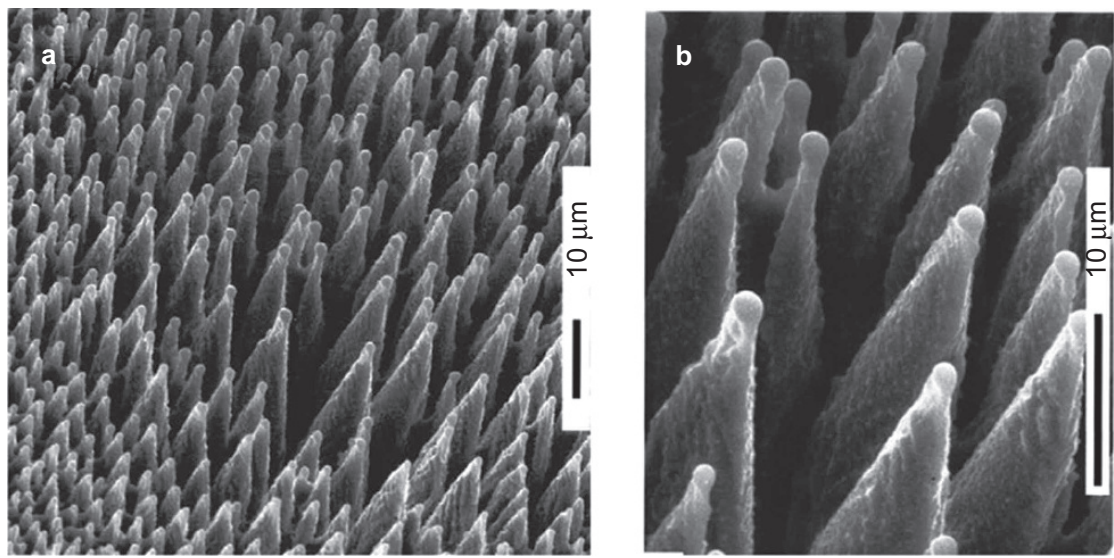

Figure 5 (a) Array of sharp conical spikes produced on Si by irradiation with $500 \mathrm{fs}$ laser pulses in $\mathrm{SF}_{6}$ gas. (b) Magnified image of (a). ${ }^{58}$ Reproduced with permission from AIP. ( 1998 by the American Institute of Physics. 


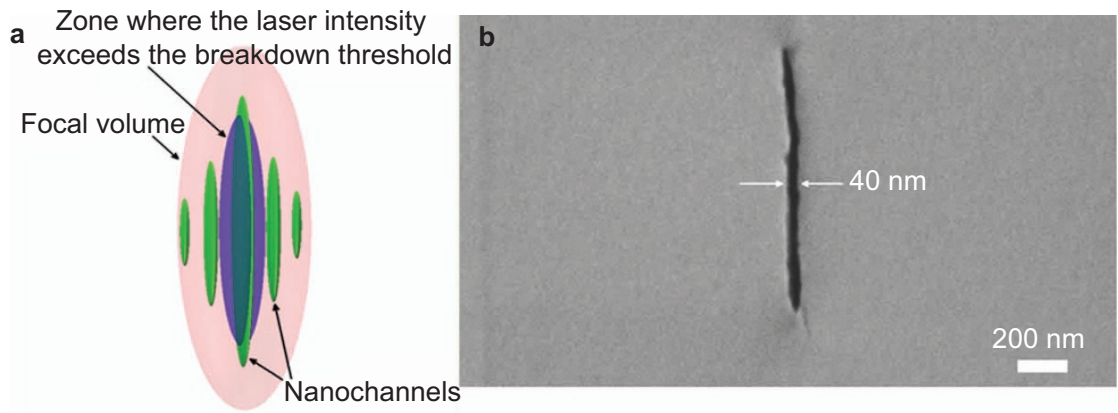

Figure 6 (a) Schematic diagram of the concept employed to realize the formation of a narrow channel width far beyond the optical diffraction limit. (b) Cross-sectional SEM micrograph of a nanochannel fabricated in porous glass. ${ }^{37}$ Reproduced with permission from RSC. ( 2013 by the Royal Society of Chemistry. SEM, scanning electron microscopy.

Figure $8 \mathrm{~b}$ shows that portions of the polymerized lines written by the activation beam alone have a relatively large width. To reduce the width of the lines, another laser beam (the deactivation beam) is operated at the same wavelength, but in the continuous wave mode, and is superimposed on the activation beam with a suitable lateral offset to deactivate the photoinitiator. With the deactivation beam, depletion of photopolymerization can be realized as a result of the metastable intermediate energy states of the photoinitiators. Thus, the portions of polymer lines written by both activation and deactivation beams are much thinner than those obtained with only the activation beam. Using this technique, a fabrication resolution of ca $40 \mathrm{~nm}$, which is $c a \lambda / 20$, has been obtained in the axial direction. It is expected that with full optimization of the photoresist properties and optical configurations, resolution of $<10 \mathrm{~nm}$ will be attainable based on this approach.

The reliable nanoscale resolution and 3D fabrication capability of the TPP technique has resulted in wide attention for diverse applications, including integrated photonics (e.g., microoptics, diffraction elements, photonic crystals and metamaterials), ${ }^{76-81}$ micromechanics, ${ }^{20}$ microelectronics, ${ }^{82}$ microfluidics ${ }^{83,84}$ and biomimetics. ${ }^{85}$ For these applications, functionalization of a photoresin is frequently required, which is mainly achieved by filling the resin with various types of functional nanoparticles, such as luminescent quantum dots, noble metal nanoparticles and magnetic nanoparticles. The nanoparticles can be either synthesized prior to filling into the resin, or synthesized in situ within the resin by exposure to the femtosecond laser. Functionalization can even be achieved by filling the resin with biomaterials. Recently, Sun et al. ${ }^{86}$ demonstrated the direct femtosecond

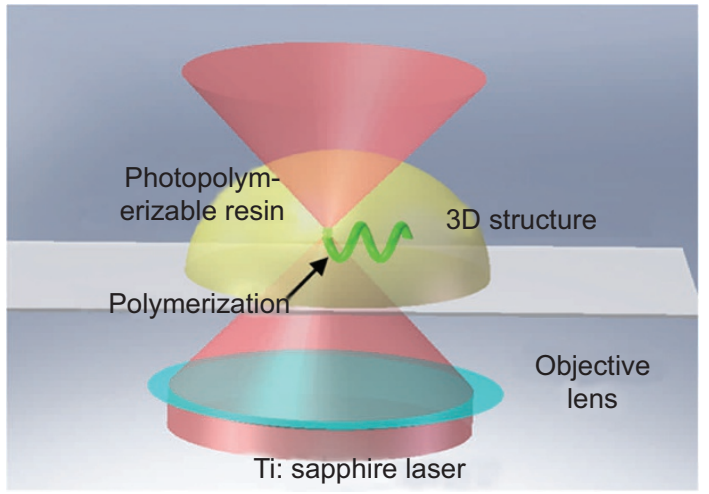

Figure 7 Schematic diagram of the fabrication process for femtosecond laserinduced TPP. 3D, three-dimensional; TPP, two-photon polymerization. laser writing of tunable microlenses by inducing photo-crosslinking in a protein (bovine serum albumin) facilitated by a photosensitizer (methylene blue). Figure 9 shows that the protein microlens becomes swollen when immersed in a buffer solution, and the degree of deformation is dependent on the $\mathrm{pH}$ of the buffer. Thus, for a protein lens with a diameter of $40 \mu \mathrm{m}$, the focal length can be continuously tuned over a wide range from $c a 400 \mu \mathrm{m}$ at $\mathrm{pH} 7.0$ to $c a 600 \mu \mathrm{m}$ at $\mathrm{pH}$ 13.0. Such unique protein-based microoptical devices are dynamically tunable and biocompatible.

\section{D processing of transparent materials}

The section on 'Characteristics of ultrafast laser processing' noted that irradiation of focused ultrafast laser pulses to transparent materials can lead to a variety of phase and structural changes in a spatially selective manner. This opens up new avenues for the fabrication of a variety of integrated microdevices for applications such as integrated photonics, optofluidics, optomechanics, optoelectronics and biomedicines. The successful construction of integrated microdevices is mainly achieved using two approaches: writing of optical waveguides along $3 \mathrm{D}$ paths by locally altering the refractive index and the formation of hollow microfluidic structures by local enhancement of the etch rate, which facilitates subsequent removal of the material in the modified region by wet chemical etching. In most cases, the direct writing scheme is adopted because it offers the maximum degree of flexibility in terms of the length and geometry of the fabricated structures. There are several review articles already available on femtosecond laser $3 \mathrm{D}$ processing in glass for optofluidic and photonic applications, and on the writing of passive and active waveguide devices in optical crystals and ceramics. ${ }^{15,16,43,44,87,88}$ Therefore, here we focus on the latest advances in $3 \mathrm{D}$ materials processing with femtosecond laser pulses for novel optofluidic and integrated photonic applications.

Optical waveguides are building blocks for various photonic devices such as beam splitters and couplers, ${ }^{89}$ Bragg grating waveguides, ${ }^{90}$ Mach-Zender interferometers, ${ }^{91}$ waveguide lasers in either glass or crystalline materials ${ }^{92-94}$ and nonlinear optical waveguide devices. ${ }^{95}$ Optical waveguides can be easily inscribed in transparent materials such as glass, crystalline materials and polymers by inducing permanent refractive index changes in the focal volumes of tightly focused femtosecond laser pulses. ${ }^{44}$ The waveguides are typically written in transparent materials using a transverse writing scheme, where the sample is translated perpendicular to the beam propagation direction. This unique capability enables waveguides of arbitrary lengths and 3D geometries to be written, and is thus particularly suitable for both photonic and optofluidic integration applications. In addition, the 

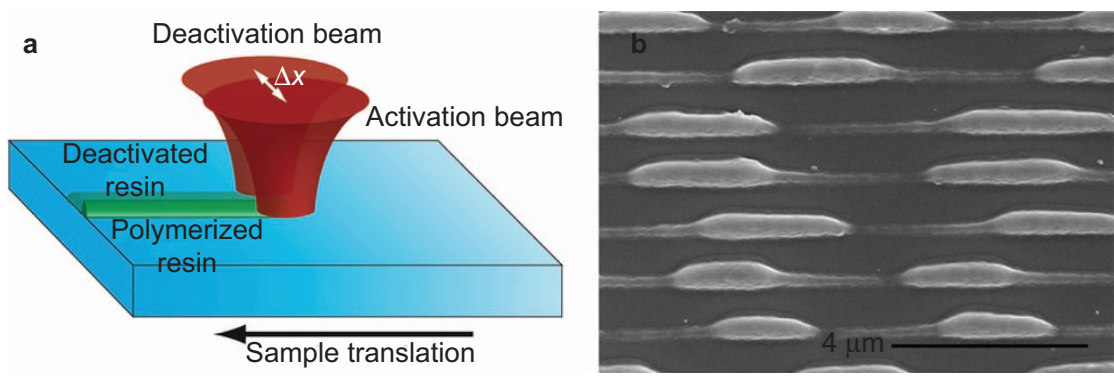

Figure 8 (a) Schematic diagram of the concept for photoinduced deactivation of photopolymerization. The excitation and deactivation beams are both focused in the prepolymer resin with a lateral separation of $\Delta x$. The substrate is translated perpendicular to the separation axis to fabricate polymer lines. (b) Plan-view SEM image of lines written with offset $(\Delta x>0), 200 \mathrm{fs}$ excitation pulses and 50 ps deactivation pulses. When the deactivation beam was chopped, the linewidth was significantly thicker. ${ }^{75}$ Reproduced with permission from AAAS. (๑2009 by the American Association for the Advancement of Science. SEM, scanning electron microscopy.

cross-sectional shape of the waveguides written with femtosecond lasers can be tailored with beam shaping techniques, which is also difficult to realize with traditional lithographic technology. ${ }^{73,96-98}$ Currently, waveguides written in glass using femtosecond lasers have typical propagation losses in the order of $0.5-1.0 \mathrm{~dB} \mathrm{~cm}^{-1}$, which is sufficiently low for most microchip devices.

Optical waveguides written using femtosecond lasers have been widely used for construction of optofluidic systems, waveguide lasers and astrophotonic devices. ${ }^{99}$ Recently, 3D waveguides written with femtosecond lasers have found important applications in quantum circuits to replace the bulk optics in complex quantum optical systems. ${ }^{100,101}$ Crespi et al. ${ }^{101}$ have constructed a multimode interferometer with femtosecond laser written waveguides for photonic boson sampling. To realize an arbitrary $5 \times 5$ mode transformation, the optical network must consist of a large amount of discrete elements such as beamsplitters and phase shifters, as shown in Figure 10a. In contrast, a waveguide-based interferometer entirely written in glass with a femtosecond laser, a 3D schematic representation of which is shown in Figure 10b, is able to provide the same functionality as a large interferometer. Moreover, the on-chip

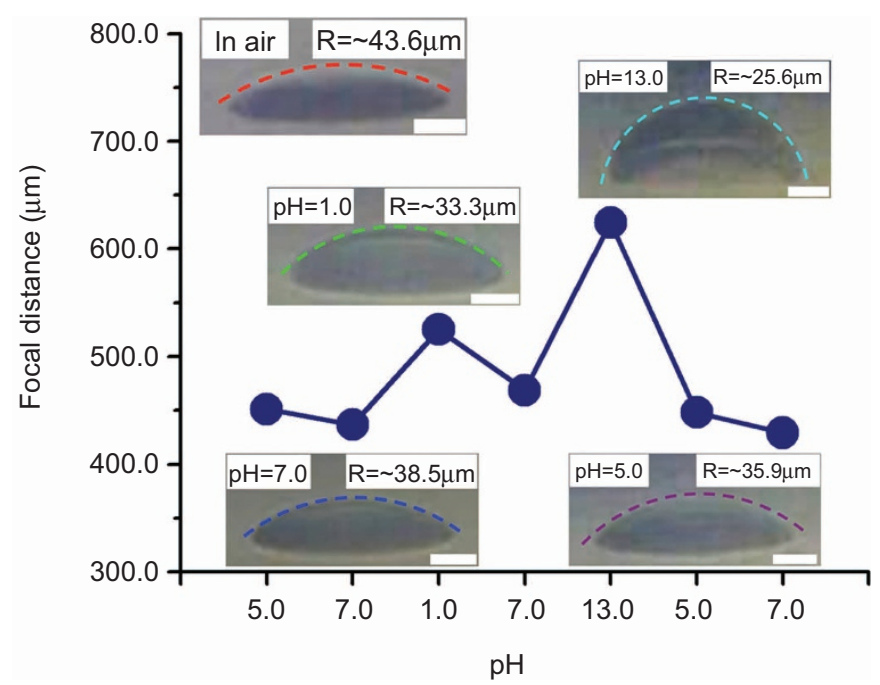

Figure 9 Focal distance of a protein microlens as a function of $\mathrm{pH}$. The inset SEM images show the protein microlens after change in the $\mathrm{pH}$. Note: the initial radius and height of the lens (fabricated at $\mathrm{pH} 7.4$ ) were 20 and $5 \mathrm{~mm}$, respectively. ${ }^{86}$ Reproduced with permission from Wiley. (C2012 by John Wiley \& Sons, Inc. SEM, scanning electron microscopy. interferometer is more robust due to the inherently stable integrated waveguide structures. It is expected that scaling up of such systems will benefit quantum computation, quantum communication and high-precision measurement.

Femtosecond lasers can also be used to fabricate microfluidic structures in glass materials by spatially selective precision modification inside glass materials through nonlinear optical absorption. Currently, there are two main strategies for this application. The first strategy employs femtosecond laser modification inside glass or crystal materials followed by wet chemical etching. ${ }^{102,103}$ The other strategy is to perform femtosecond laser 3D drilling from the rear surface of the glass in contact with distilled water, by which the water introduced into the microchannel can help to remove the ablated debris. ${ }^{104,105}$ The former approach has advantages that include larger scale microfluidic structures and improved smoothness on inner wall, whereas the latter approach allows for the fabrication of 3D microchannels in an almost limitless variety of materials with smaller channel diameters. In particular, microfluidic channels with arbitrary lengths have recently been produced in porous glass immersed in water using water-assisted femtosecond laser drilling. ${ }^{106}$ This approach has also enabled the fabrication of $c a 40 \mu \mathrm{m}$ long nanofluidic channels with widths of $c a$ $40 \mathrm{~nm},{ }^{37,107}$ as shown in Figure 6.

The unique ability of direct femtosecond laser writing to simultaneously alter the chemical and optical properties of bulk glass materials provides new avenues to fabricate a variety of integrated microfluidic and optofluidic microsystems/devices such as microfluidic lasers, ${ }^{108}$ nanoaquariums to observe and determine the functions of living organisms, ${ }^{109,110}$ optofluidic sensors with various functions including liquid concentration analysis, ${ }^{11,112}$ single-cell detection and manipulation systems ${ }^{113-115}$ and devices for the rapid screening of algae populations. ${ }^{116}$ The latest progress is aimed at realizing an optofluidic sensor by coating the inner wall of a microfluidic channel with a polymer, as illustrated in Figure 11a. ${ }^{112}$ Glass materials typically have refractive indices higher than most fluids, so that light propagating along a microfluidic channel filled with liquid sample will experience significant loss. In contrast, such a scheme can provide good sensitivity because of the long interaction length between the light and the sample. This problem was addressed by Hanada et al., ${ }^{112}$ where the inner walls of prefabricated microchannels in glass were coated with a polymer of which the refractive index was lower than water, as shown in Figure 11b. This leads to the low-loss propagation of light in microfluidic channels by total internal reflection at the liquid/polymer surface. This improvement realized a sensitivity of $200 \mathrm{nM}$ for glucose$\mathrm{D}$ as a sample analyte. ${ }^{112}$ 
a $\ln$

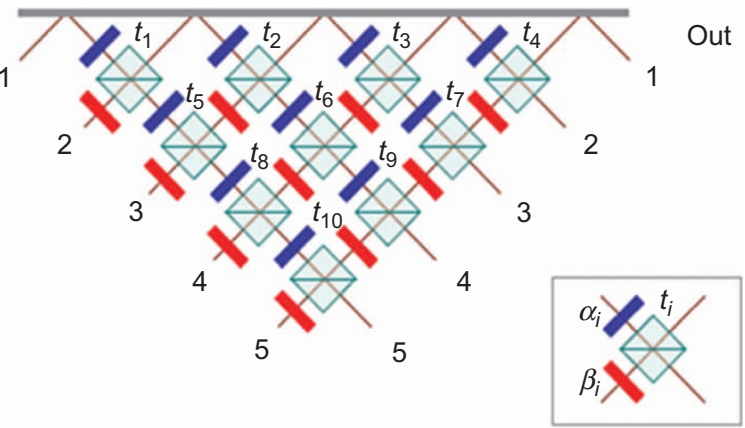

b

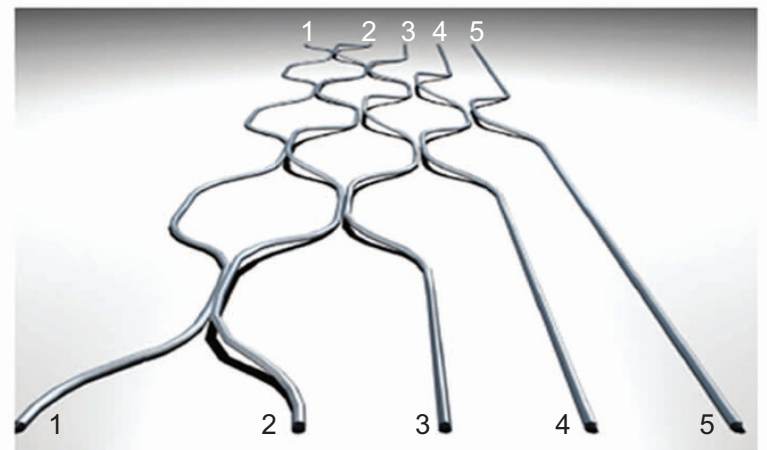

Figure 10 (a) Layout of a multimode interferometer for realization of an arbitrary $5 \times 5$ mode transformation using a network of beamsplitters with different transmissivities. (b) The same interferometer realized in a glass substrate using a laser writing technique. ${ }^{101}$ Reproduced with permission from NPG. (C2013 by Nature Publishing Group.

Femtosecond lasers have also been used to produce various types of microoptical components in glass. Microoptical mirrors and lenses can be fabricated using the air/glass interface created inside glass by direct femtosecond laser writing and subsequent chemical etching. ${ }^{117,118}$ For such optical applications, the surface roughness on the as-etched inner walls is still too high, which creates significant loss and distortion of the wavefront. The surface roughness can be dramatically improved with an additional post-annealing process at a temperature slightly lower than the melting point of glass, which induces surface reflow for surface smoothening without causing deformation to the fabricated structures. ${ }^{119}$ In addition, the fabrication of polarization-sensitive optics with femtosecond lasers is now undergoing rapid growth. This fabrication is achieved by arranging the distributions and orientations of nanogratings written in glass using polarization controlled femtosecond laser pulses. ${ }^{39,56}$ This method has enabled the production of devices such as diffraction gratings, polarization and optical vortex converters. ${ }^{120,121}$

\section{COMMERCIAL APPLICATIONS}

Ultrafast laser ablation was applied to the repair of chromium-onfused-silica photomasks for deep ultraviolet photolithography in the field of semiconductor manufacturing at IBM's mask-making facility. ${ }^{122}$ Pulses of $100 \mathrm{fs}$ with a wavelength of $266 \mathrm{~nm}$ realized a spatial resolution better than $100 \mathrm{~nm} .{ }^{123}$ Concurrently, the first application of ultrafast lasers to mass production processes was drilling inkjet nozzles with specific shapes by picosecond laser ablation. ${ }^{122}$

Approximately 10 years ago, ultrafast laser processing showed potential to satisfy many requirements in the automotive industry, including miniaturization, high precision, high quality, applicability to a diversity of materials, variety of variants, smaller lots and cost-effectiveness. For a

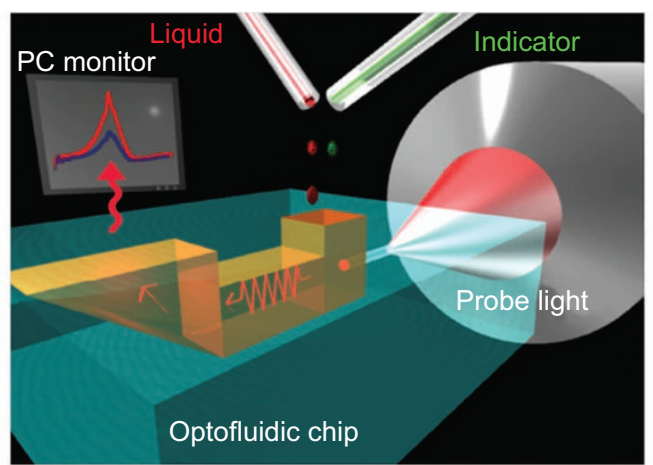

b

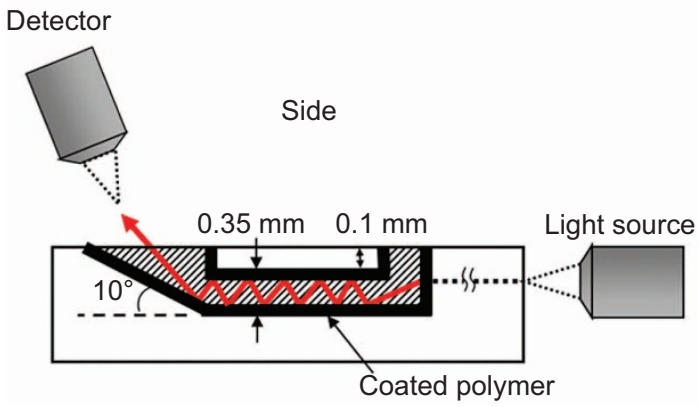

Figure 11 (a) Schematic diagram of an optofluidic chip for liquid concentration measurement. The optofluidic chip is filled with liquid and a white light probe is coupled to the entrance facet of the waveguide. The light transmitted by the waveguide propagates inside the microfluidic channel and is then reflected at the tilted wall of the left reservoir, where the absorption spectra of liquids are measured. (b) Design of the optofluidic chip as illustrated in (a). Note that the inner surface of microchannel is coated with polymer. ${ }^{112}$ Reproduced with permission from RSC. (C)2012 by the Royal Society of Chemistry.

example, the structuring of small grooves into the surface of the cylinder wall of a combustion engine by design ensured complete distribution of lubricant along the piston wall and reduced the friction losses of the engine. A more beneficial point of this structure is a reduction in the amount of lubricant injected into the engine, which significantly reduces particle emissions and extends the system lifetime. This technique has been implemented by various car manufacturers including serial production and racing sports manufacturers. ${ }^{124}$ Since 2007 , picosecond laser trimming has been used to produce exhaust gas sensors, as shown in Figure 12a, at the Robert Bosch plant in Bamberg, Germany. ${ }^{125}$ These sensors have a special ceramic layer and can measure the exhaust gas properties faster and more precisely than conventional sensors. Such sensors enable emissions to be reduced by optimizing combustion control. Furthermore, since 2009, picosecond lasers have been used to fabricate diesel engine injectors, where drain grooves are microstructured around a high-pressure passage using picosecond laser pulses, as shown in Figure 12b. ${ }^{125}$ The structured drainage grooves allow a tight system, even at pressures up to 2000 bar. As a result, diesel injection systems have become more reliable, powerful and environmental friendly.

In 2011, black Si, which is produced by femtosecond laser irradiation in a halogen atmosphere (Figure 5), was applied to produce commercial photovoltaic Si solar cells by SiOnyx Inc. ${ }^{126}$ Average efficiencies as high as $16.9 \%$ were achieved for $150 \mu \mathrm{m}$ thick multicrystalline Si that was $20 \%$ thinner than conventionally used wafers; a cost reduction of $10 \%-15 \%$ was realized. Ultrafast lasers are also promising tools for scribing and patterning in the manufacture of photovoltaic solar cells based on copper indium gallium (di)selenide (CIGS) or 

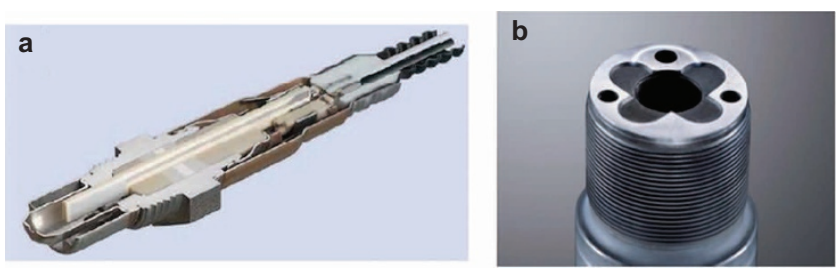

Figure 12 (a) Cross-section of exhaust gas sensor produced by picosecond laser trimming. (b) Groove around a high pressure coupling to avoid discharge of fuel. ${ }^{125}$ Reproduced with permission from JLPS. ( 02010 by the Japan Laser Processing Society.

copper indium selenide. ${ }^{127}$ For patterning, it is necessary to isolate the back contact (Mo) between cells ( $\mathrm{P} 1$ process), selectively remove the CIGS layer from the top of the back contact layer ( $\mathrm{P} 2$ process) and selectively remove the absorber and front contact (transparent conductive oxide) layers ( $\mathrm{P} 3$ process). Conventional nanosecond lasers are widely used for $\mathrm{P} 1$ processes, whereas $\mathrm{P} 2$ and $\mathrm{P} 3$ processes are typically processed using mechanical scribing tools. This is due to the structure of CIGS photovoltaic solar cells. Specifically, when fabricating CIGS photovoltaic solar cells, a metallic back contact is first deposited on the glass substrate to form a broadband reflective layer at the rear surface of the module. The absorber and front contact transparent conductive oxide layers are then deposited on top, and these layers must be processed from the front to prevent damage to the metallic back contact. Picosecond laser processing can replace mechanical scribing for the P2 and P3 processes because it does not cause thermal damage to the underlying layers. However, its adoption is currently limited because the process is immature and more expensive than conventional nanosecond laser processing.

Meanwhile, ultrafast lasers perform scribing and cutting of glass with not only high quality edge, but also flexible geometries. These features have a widespread impact on numerous applications, including ultrathin to hardened glass, which have led to a breakthrough for ultrafast lasers in the mass production of displays for cell phones and

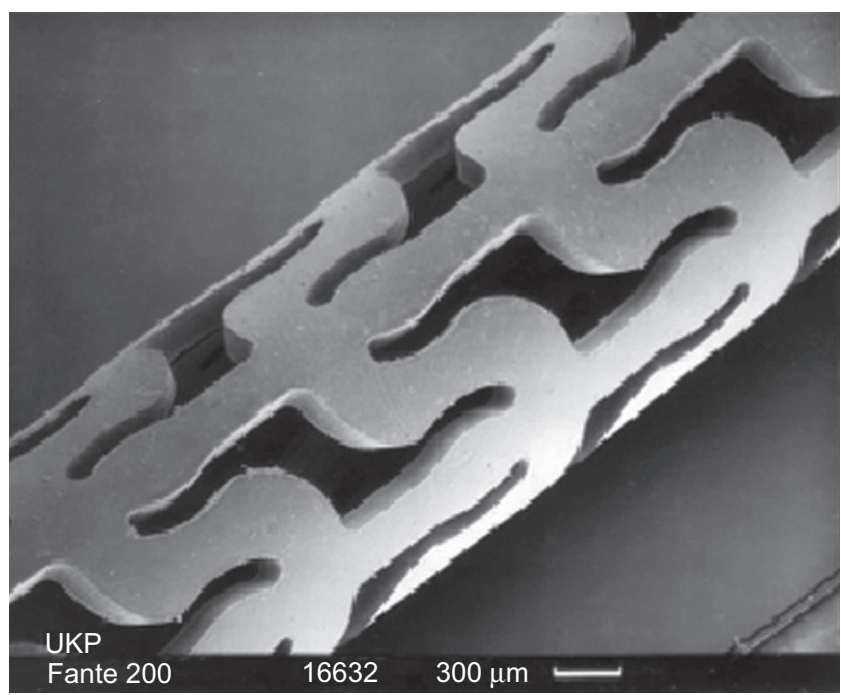

Figure 13 Prototype medical stent fabricated from a bioresorbable polymer. ${ }^{129}$ Reproduced with permission from SPIE. (C2000 by SPIE-the International Society for Optics and Photonics. tablet computers. ${ }^{128}$ Ultrafast laser patterning is also expected to be used for the manufacture of organic light emitting diode displays.

One practical application of ultrafast lasers in the medical field is the fabrication of coronary stents, which are used for the minimally invasive treatment of arteriosclerosis as an alternative to bypass operations. Stents are typically fabricated from stainless steel or shape memory alloys. However, it is necessary to post-process these materials chemically to realize the required properties. In addition, there have been some medical problems with stents, such as the risk of restenosis and limited biocompatibility. To achieve better biocompatibility, $\mathrm{Mg}$ based alloys or special biopolymers should be used. However, these materials also suffer from problems in stent production, in that they require a poorly established post-processing technique and they react strongly to thermal loads. Ultrafast laser micromachining can overcome these problems since it produces little debris as well as little HAZ. Figure 13 shows a medical stent fabricated from a bioresorbable polymer using femtosecond laser ablation without post-processing ${ }^{129}$ that is currently ready for practical use.

Another application in the medical field is laser-assisted in situ keratomileusis, commonly referred to as LASIK, which is a refractive surgery used to correct myopia, hyperopia, and astigmatism using a laser. ${ }^{130}$ Figure 14 shows an optical microscope image of a cornea in which femtosecond laser irradiation has created a flap and a lens body by making cuts inside the cornea. A system equipped with a femtosecond laser to form corneal flaps (Femto-LASIK) is now commercially available, and is more precise and predictable than a conventional mechanical microkeratome. ${ }^{131}$

\section{SUMMARY AND OUTLOOK}

Ultrafast laser processing has fundamentally changed the way of materials microprocessing with lasers. This approach provides unique advantages such as the suppression of thermal diffusion, nonlinear multiphoton excitation of carriers, deterministic optical breakdown threshold, internal modification of transparent materials and reproducible nanoscale resolution. It has become a ubiquitous micromachining tool for various materials including metals, semiconductors, ceramics, glass, crystals, polymers and even soft materials such as biotissues. When being applied for surface structuring, ultrafast laser processing induces novel properties in terms of friction, adhesion, optical absorption and/or reflection, and hydrophobicity. Scanning of tightly focused ultrafast laser pulses in transparent materials enables the formation of 3D micro-/nanostructures with limitless geometries and configurations, which makes it possible to produce functional components and devices for integrated optics and lab-on-a-chip applications. Besides fundamental research, ultrafast lasers have also been employed for several practical and industrial applications, such as photomask repairs, ink nozzle drilling and medical stent fabrication. These lasers are now also used in the electronics industry for scribing, patterning and texturing of glass and semiconductors (e.g., photovoltaic cells and light emitting diode displays).

The past efforts in this research field have mostly been in three directions; uncovering new physical mechanisms, developing new fabrication techniques and improving performance, and extending the application range. This general trend will continue in the future, although the latter direction may be more emphasized. On the mechanism investigation side, recent interest has been focused on femtosecond laser-induced non-ablative processes, such as nanograting formation at critical optical intensity, and phase and structural changes in high pressure nano-environments. Understanding these physical processes requires the diagnosis of ultrafast plasma dynamics 


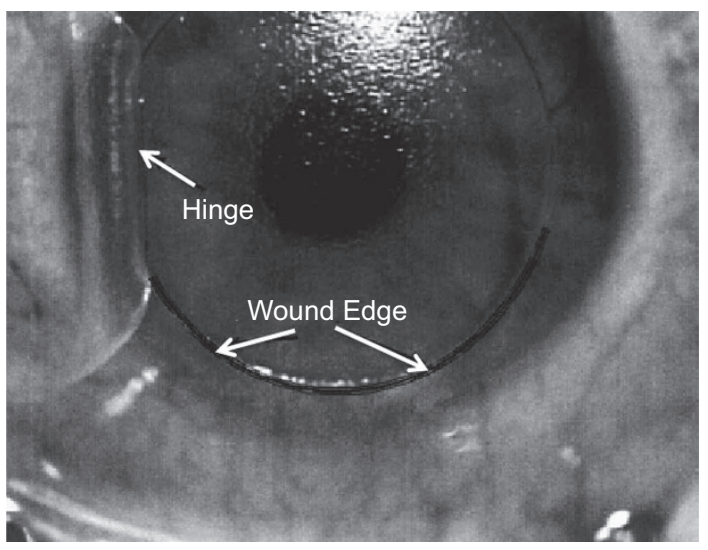

Figure 14 Optical microscope image of a cornea in which femtosecond laser irradiation has formed a flap and a lens body by making cuts inside the cornea. ${ }^{130}$ Reproduced with permission from Elsevier. @2004 by Elsevier.

inside transparent materials with micron or submicron spatial resolution and few- or subfemtosecond temporal resolution. On the technical development side, significant progress has been achieved in the development of novel ultrafast laser systems. The replacement of Ti:sapphire crystals with fibers or thin disks has not only led to high average powers (i.e., intense ultrafast laser pulses at high repetition rates) that will allow direct laser writing at speeds leveling the order of tens of meters per second, but will also make the ultrafast laser systems more robust and cost-effective. ${ }^{132,133}$ The rich variety of parameters provided by ultrafast laser pulses has allowed femtosecond laser beam shaping to be extensively investigated. Early efforts were mainly focused on spatial or temporal shaping of ultrafast laser pulses, whereas recent interest has shifted to simultaneous spatiotemporal manipulation of the femtosecond laser pulses, which has enabled modification of the peak intensity distribution in the focal area, ${ }^{134}$ the elimination of self-focusing, ${ }^{135,136}$ high-efficiency 3D fabrication $^{137}$ and induction of the nonreciprocal writing effect. ${ }^{138}$ In addition, manipulation of the femtosecond laser pulse polarization has enabled the fabrication of polarization-sensitive optical devices and nanofluidic channels in glass. Beam shaping effects have also been exploited in TPP, aimed at either high-speed parallel processing or subdiffraction-limited nanostructuring. Continuous improvement in understanding the physical mechanisms and advances in the development of fabrication techniques will help accelerate the widespread use of ultrafast laser processing over a broad range of applications, including integrated optics and photonics, optofluidics, optomechanics, optoelectronics, surface micro- and nanostructuring, glass welding and tissue engineering. ${ }^{139}$

1 Srinivasan R, Sutcliffe E, Braren B. Ablation and etching of polymethylmethacrylate by very short (160 fs) ultraviolet (308 nm) laser pulses. Appl Phys Lett 1987; 51: 1285-1287.

2 Küper S, Stuke M. Femtosecond UV excimer laser ablation. Appl Phys B 1987; 44: 199-204.

3 Rudd JV, Korn G, Kane S, Squier J, Mourou G et al. Chirped-pulse amplification of 55fs pulses at a $1-\mathrm{kHz}$ repetition rate in a $\mathrm{Ti}-\mathrm{Al}_{2} \mathrm{O}_{3}$ regenerative amplifier. Opt Lett 1993; 18: 2044-2046.

4 Momma C, Chichkov BN, Nolte S, von Alvensleben F, Tünnermann A et al. Short-pulse laser ablation of solid targets. Opt Commun 1996; 129: 134-142.

5 Yanik MF, Cinar H, Cinar HN, Chisholm AD, Jin Y et al. Neurosurgery: functional regeneration after laser axotomy. Nature 2004; 432: 822 .

6 Bärsch N, Körber K, Ostendorf A, Tönshoff KH. Ablation and cutting of planar silicon devices using femtosecond laser pulses. Appl Phys A 2003; 77: 237-242.
7 Nakata $\mathrm{Y}$, Okada T, Maeda M. Fabrication of dot matrix, comb, and nanowire structures using laser ablation by interfered femtosecond laser beams. Appl Phys Lett 2002; 81: 4239-4241.

8 Küper S, Stuke M. Ablation of polytetrafluoroethylene (Teflon) with femtosecond UV excimer laser pulses. Appl Phys Lett 1989; 54: 4-6.

9 Küper S, Stuke M. Ablation of UV-transparent materials with femtosecond UV excimer laser pulses. Microelectron Eng 1989; 9: 475-480.

10 Krüger J, Kautek W. Femtosecond-pulse visible laser processing of transparent materials. App/ Surf Sci 1996; 96-98: 430-438.

11 Davis KM, Miura K, Sugimoto N, Hirao K. Writing waveguides in glass with a femtosecond laser. Opt Lett 1996; 21: 1729-1731.

12 Glezer EN, Milosavljevic M, Huang L, Finlay RJ, Her TH et al. Three-dimensional optical storage inside transparent materials. Opt Lett 1996; 21: 2023-2025.

13 Watanabe W, Sowa S, Tamaki T, Itoh K, Nishii J. Three-dimensional waveguides fabricated in poly(methyl methacrylate) by a femtosecond laser. Jpn J Appl Phys 2006; 45: L765-L767.

14 Hanada Y, Sugioka K, Midorikawa K. UV waveguides light fabricated in fluoropolymer CYTOP by femtosecond laser direct writing. Opt Express 2010; 18: 446-450.

15 Itoh K, Watanabe W, Nolte S, Schaffer CB. Ultrafast processes for bulk modification of transparent materials. MRS Bull 2006; 31: 620-625.

16 Sugioka K, Cheng Y. Femtosecond laser processing for optofluidic fabrication. Lab Chip 2012; 12: 3576-3589.

17 Kawata S, Sun HB, Tanaka T, Takada K. Finer features for functional microdevices. Nature 2001; 412: 697-698.

18 Tan DF, Li Y, Qi FJ, Yang H, Gong QH et al. Reduction in feature size of two-photon polymerization using SCR500. Appl Phys Lett 2007; 90: 071106.

19 Sun HB, Matsuo S, Misawa H. Three-dimensional photonic crystal structures achieved with two-photon-absorption photopolymerization of resin. Appl Phys Lett 1999; 74: 786-788.

20 Maruo S, Ikuta K, Korogi H. Submicron manipulation tools driven by light in a liquid. Appl Phys Lett 2003; 82: 133-135.

21 Xu BB, Zhang YL, Xia H, Dong WF, Ding $\mathrm{H}$ et al. Fabrication and multifunction integration of microfluidic chips by femtosecond laser direct writing. Lab Chip 2013; 13: 1677-1690.

22 Reif J, Costache F, Henyk M, Pandelov SV. Ripples revisited: non-classical morphology at the bottom of femtosecond laser ablation craters in transparent dielectrics. Appl Surf Sci 2002; 197-198: 891-895.

23 Wu QH, Ma YR, Fang RC, Liao Y, Yu QX et al. Femtosecond laser-induced periodic surface structure on diamond film. Appl Phys Lett 2003; 82: 1703-1705.

24 Rudolph P, Kautek W. Composition influence of non-oxidic ceramics on selfassembled nanostructures due to fs-laser irradiation. Thin Solid Films 2004; 453454: 537-541.

25 Miyaji G, Miyazaki K. Ultrafast dynamics of periodic nanostructure formation on diamondlike carbon films irradiated with femtosecond laser pulses. Appl Phys Lett 2006; 89: 191902.

26 Carey JE, Crouch $\mathrm{CH}$, Shen M, Mazur E. Visible and near-infrared responsivity of femtosecond-laser microstructured silicon photodiodes. Opt Lett 2005; 30: 17731775.

27 Baldacchini T, Carey JE, Zhou M, Mazur E. Superhydrophobic surfaces prepared by microstructuring of silicon using a femtosecond laser. Langmuir 2006; 22: 49174919.

28 Arai A, Bovatsek J, Yoshino F, Liu Z, Cho GC et al. Fiber chirped pulse amplification system for micromachining. Proc SPIE 2006; 6343: 63430S

29 Kleinbauer J, Eckert D, Weiler S, Sutter DH. 80 W ultrafast CPA-free disk laser. Proc SPIE 2008; 6871: 68711B.

30 Marchese SV, Baer CR, Engqvist AG, Hashimoto S, Maas DJ et al. Femtosecond thin disk laser oscillator with pulse energy beyond the 10-microjoule level. Opt Express 2008; 16: 6397-6407

31 Fan WS, Storz R, Tom HW, Bokor J. Electron thermalization in gold. Phys Rev B 1992 46: 13592-13595.

32 Wellershoff SS, Hohlfeld J, Güdde J, Matthias E. The role of electron-phonon coupling in femtosecond laser damage of metals. Appl Phys A 1999; 69: S99-S107.

33 Bloembergen N. A brief history of light breakdown. J Nonlinear Opt Phys 1997; 6: 377-385.

34 Stuart BC, Feit MD, Rubenchik AM, Shore BW, Perry MD. Laser-induced damage in dielectrics with nanosecond to subpicosecond pulses. Phys Rev Lett 1995; 74: 2248-2251.

35 Stuart BC, Feit MD, Herman S, Rubenchik AM, Shore BW et al. Nanosecond-tofemtosecond laser-induced breakdown in dielectrics. Phys Rev B 1996; 53: 17491761.

36 Joglekar AP, Liu H, Meyhöfer E, Mourou G, Hunt AJ. Optics at critical intensity: applications to nanomorphing. Proc Natl Acad Sci USA 2004; 101: 5856-5861.

37 Liao Y, Cheng Y, Liu CN, Song JX, He F et al. Direct laser writing of sub-50 nm nanofluidic channels buried in glass for three-dimensional micro-nanofluidic integration. Lab Chip 2013; 13: 1626-1631.

38 Kanehira S, Si J, Qiu J, Fujita K, Hirao K. Periodic nanovoid structures via femtosecond laser irradiation. Nano Lett 2005; 5: 1591-1595.

39 Shimotsuma Y, Kazansky PG, Qiu JR, Hirao K. Self-organized nanogratings in glass irradiated by ultrashort light pulses. Phys Rev Lett 2003; 91: 247405.

40 Liu Y, Shimizu M, Zhu B, Dai Y, Qian B et al. Micromodification of element distribution in glass using femtosecond laser irradiation. Opt Lett 2009; 34: 136-138. 
41 Cheng Y, Sugioka K, Masuda M, Shihoyama K, Toyoda K et al. Optical gratings embedded in photosensitive glass by photochemical reaction using a femtosecond laser. Opt Express 2003; 11: 1809-1816.

42 Kazansky PG, Yang W, Bricchi E, Bovatsek J, Arai A et al. "Quill" writing with ultrashort light pulses in transparent materials. Appl Phys Lett 2007; 90: 151120.

43 Gattass RR, Mazur E. Femtosecond laser micromachining in transparent materials. Nature Photon 2008; 2: 219-225.

44 Osellame R, Hoekstra HJ, Cerullo G, Pollnau M. Femtosecond laser microstructuring: an enabling tool for optofluidic lab-on-chips. Laser Photonics Rev2011; 5: 442-463.

45 Chichkov BN, Momma C, Nolte S, von Alvensleben F, Tünnermann A. Femtosecond, picosecond and nanosecond laser ablation of solids. App/ Phys A 1996; 63: 109115.

46 Emmony DC, Howson RP, Willis LJ. Laser mirror damage in germanium at $10.6 \mu \mathrm{m}$. Appl Phys Lett 1973; 23: 598-600.

47 Jain AK, Kulkarni VN, Sood DK, Uppal JS. Periodic surface ripples in laser-treated aluminum and their use to determine absorbed power. J Appl Phys 1981; 52: 4882 4884.

48 Keilmann $\mathrm{F}$, Bai YH. Periodic surface structures frozen into $\mathrm{CO}_{2}$ laser-melted quartz. Appl Phys A 1982; 29: 9-18.

49 Sakabe S, Hashida M, Tokita S, Namba S, Okamuro K. Mechanism for self-formation of periodic grating structures on a metal surface by a femtosecond laser pulse. Phys Rev B 2009; 79: 033409.

50 Hashida M, Fujita M, Tsukamoto M, Semerok AF, Gobert 0 et al. Femtosecond laser ablation of metals: precise measurement and analytical model for crater profiles. Proc SPIE 2003: 4830: 452-457.

51 Yasumaru N, Miyazaki K, Kiuchi J. Femtosecond-laser-induced nanostructure formed on hard thin films of TiN and DLC. Appl Phys A 2003; 76: 983-985.

52 Borowiec A, Hauge HK. Subwavelength ripple formation on the surfaces of compound semiconductors irradiated with femtosecond laser pulses. App/ Phys Lett 2003; 82 4462-4464.

53 Costache F, Henyk M, Reif J. Surface patterning on insulators upon femtosecond laser ablation. App/ Surf Sci 2003; 208-209: 486-491.

54 Le Harzic R, Dörr D, Sauer D, Neumeier M, Epple M et al. Large-area, uniform, highspatial-frequency ripples generated on silicon using a nanojoule-femtosecond laser at high repetition rate. Opt Lett 2011; 36: 229-231.

55 Wang $\mathrm{C}$, Huo H, Johnson M, Shen M, Mazur E. The thresholds of surface nano-/micromorphology modifications with femtosecond laser pulse irradiations. Nanotechnology 2010; 21: 075304.

56 Bhardwaj VR, Simova E, Rajeev PP, Hnatovsky C, Taylor RS et al. Optically produced arrays of planar nanostructures inside fused silica. Phys Rev Lett 2006; 96: 057404.

57 Miyaji G, Miyazaki K. Origin of periodicity in nanostructuring on thin film surfaces ablated with femtosecond laser pulses. Opt Express 2008; 16: 16265-16271.

58 Her TH, Finlay RJ, Wu C, Deliwala S, Mazur E. Microstructuring of silicon with femtosecond laser pulses. Appl Phys Lett 1998; 73: 1673-1675.

59 Younkin R, Carey JE, Mazur E, Levinson JA, Friend CM. Infrared absorption by conical silicon microstructures made in a variety of background gases using femtosecondlaser pulses. J Appl Phys 2003; 93: 2626-2629.

60 Wang $\mathrm{F}$, Chen $\mathrm{C}$, He H, Liu S. Analysis of sunlight loss for femtosecond lase microstructured silicon and its solar cell efficiency. App/ Phys A 2011; 103: 977982.

61 Zorba V, Stratakis E, Barberoglou M, Spanakis E, Tzanetakis P et al. Biomimetic artificial surfaces quantitatively reproduce the water repellency of a lotus leaf. Adv Mater 2008; 20: 4049-4054.

62 Nayak BK, Gupta MC, Kolasinski KW. Spontaneous formation of nanospiked microstructures in germanium by femtosecond laser irradiation. Nanotechnology 2007; 18: 195302

63 Nakashima S, Sugioka K, Midorikawa K. Enhancement of resolution and quality of nano-hole structure on GaN substrates using the second-harmonic beam of nearinfrared femtosecond laser. Appl Phys A 2010; 101: 475-481.

64 Nakashima S, Sugioka K, Ito T, Takai H, Midorikawa K. Fabrication of high-aspectratio nanohole arrays on GaN surface by using wet-chemical-assisted femtosecond laser ablation. J Laser Micro/Nanoeng 2011; 6: 15-19.

65 Chimmalgi A, Choi TY, Grigoropoulos CP, Komvopoulos K. Femtosecond lase aperturless near-field nanomachining of metals assisted by scanning probe microscopy. Appl Phys Lett 2003; 82: 1146-1148.

66 Lin Y, Hong MH, Wang WJ, Law YZ, Chong TC. Sub-30 nm lithography with near-field scanning optical microscope combined with femtosecond laser. Appl Phys A 2005; 80: 461-465

67 Atanasov PA, Takada H, Nedyalkov NN, Obara M. Nanohole processing on silicon substrate by femtosecond laser pulse with localized surface plasmon polariton. App Surf Sci 2007; 253: 8304-8308.

68 Eversole D, Luk'yanchuk B, Ben-Yakar A. Plasmonic laser nanoablation of silicon by the scattering of femtosecond pulses near gold nanospheres. Appl Phys A 2007; 89: 283-291.

69 Maruo S, Nakamura O, Kawata S. Three-dimensional microfabrication with twophoton-absorbed photopolymerization. Opt Lett 1997; 22: 132-134.

70 Cumpston BH, Ananthavel SP, Barlow S, Dyer DL, Ehrlich JE et al. Two-photon polymerization initiators for three-dimensional optical data storage and microfabrication. Nature 1999; 398: 51-54.

71 Maruo S, Fourkas JT. Recent progress in multiphoton microfabrication. Laser Photonics Rev 2008; 2: 100-111.

72 Sun HB, Tanaka T, Kawata S. Three-dimensional focal spots related to two-photon excitation. Appl Phys Lett 2002; 80: 3673-3675.
73 Cheng Y, Sugioka K, Midorikawa K, Masuda M, Toyoda K et al. Control of the crosssectional shape of a hollow microchannel embedded in photostructurable glass by use of a femtosecond laser. Opt Lett 2003; 28: 55-57.

74 Hell SW, Wichmann J. Breaking the diffraction resolution limit by stimulated emission: stimulated-emission-depletion fluorescence microscopy. Opt Lett 1994; 19: 780-782.

75 Li L, Gattass RR, Gershgoren E, Hwang H, Fourkas JT. Achieving $\lambda / 20$ resolution by one-color initiation and deactivation of polymerization. Science 2009; 324: 910-913.

76 Wu D, Niu LG, Chen QD, Wang R, Sun HB. High efficiency multilevel phase-type fractal zone plates. Opt Lett 2008; 33: 2913-2915.

77 Chen QD, Lin XF, Niu LG, Wu D, Wang WQ et al. Dammann gratings as integratable micro-optical elements created by laser micronanofabrication via two-photon photopolymerization. Opt Lett 2008; 33: 2559-2561.

78 Seet KK, Mizeikis V, Matsuo S, Juodkazis S, Misawa H. Three-dimensional spiralarchitecture photonic crystals obtained by direct laser writing. Adv Mater 2005; 17: 541-545.

79 Deubel M, von Freymann G, Wegener M, Pereira S, Busch K et al. Direct laser writing of three-dimensional photonic-crystal templates for telecommunications. Nat Mater 2004; 3: 444-447.

80 Michael SR, Christine P, Michael T, Isabelle S, von Freymann G et al. Photonic metamaterials by direct laser writing and silver chemical vapour deposition. Nat Mater 2008; 7: 543-546.

81 Gansel JK, Thiel M, Rill MS, Decker M, Bade K et al. Gold helix photonic metamaterial as broadband circular polarizer. Science 2009; 325: 1513-1515.

82 Ikegami T, Ozawa R, Stocker MP, Monaco K, Fourkas JT et al. Development of optically-driven metallic microrotors using two-photon microfabrication. J Laser Micro/Nanoeng 2013; 8: 6-10.

83 Wu D, Chen QD, Niu LG, Wang JN, Wang J et al. Femtosecond laser rapid prototyping of nanoshells and suspending components towards microfluidic devices. Lab Chip 2009; 9: 2391-2394.

84 Amato L, GuY, Bellini N, Eaton SM, Cerullo G et al. Integrated three-dimensional filter separates nanoscale from microscale elements in a microfluidic chip. Lab Chip 2012; 12: $1135-1142$

85 Zhang YL, Xia H, Kim E, Sun HB. Recent developments in superhydrophobic surfaces with unique structural and functional properties. Soft Matter 2012; 8: 1121711231.

86 Sun YL, Dong WF, Yang RZ, Meng X, Zhang L et al. Dynamically tunable protein microlenses. Angew Chem Int Ed 2012; 51: 1558-1562.

87 Chen F, Vázquez de Aldana JR. Optical waveguides in crystalline dielectric materials produced by femtosecond-laser micromachining. Laser Photonics Rev; e-pub ahead of print 17 May 2013; doi:10.1002/Ipor.201300025.

88 Ams M, Marshall GD, Dekker P, Dubov M, Mezentsev VK et al. Investigation of ultrafast laser-photonic material interactions: challenges for directly written glass photonics. IEEE J Sel Top Quant 2008; 14: 1370-1381.

89 Watanabe W, Asano T, Yamada K, Itoh K, Nishii J. Wavelength division with threedimensional couplers fabricated by filamentation of femtosecond laser pulses. Opt Lett 2003; 28: 2491-2493.

90 Martinez A, Dubov M, Khrushchev I, Bennion I. Direct writing of fibre Bragg gratings by femtosecond laser. Electron Lett 2004; 40: 1170-1172.

91 Liao Y, Xu J, Cheng Y, Zhou ZH, He F et al. Electro-optic integration of embedded electrodes and waveguides in $\mathrm{LiNbO}_{3}$ using a femtosecond laser. Opt Lett 2008; 33: 2281-2283.

92 Ams M, Dekker P, Marshall GD, Withford MJ. Monolithic $100 \mathrm{~mW}$ Yb waveguide lase fabricated using the femtosecond-laser direct-write technique. Opt Lett 2009; 34 247-249

93 Okhrimchuk AG, Shestakov AV, Khrushchev I, Mitchell J. Depressed cladding, buried waveguide laser formed in a YAG: $\mathrm{Nd}^{3+}$ crystal by femtosecond laser writing. Opt Lett 2005; 30: 2248-2250.

94 Torchia GA, Rodenas A, Benayas A, Cantelar E, Roso L et al. Highly efficient lase action in femtosecond-written $\mathrm{Nd}$ :yttrium aluminum garnet ceramic waveguides. $A p p /$ Phys Lett 2008; 92: 111103

95 Dong NN, Martínez de Mendivil J, Cantelar E, Lifante G, Vázquez de Aldana J et al. Self-frequency-doubling of ultrafast laser inscribed neodymium doped yttrium aluminum borate waveguides. Appl Phys Lett 2011; 98: 181103.

96 Osellame R, Taccheo S, Marangoni M, Ramponi R, Laporta P et al. Femtosecond writing of active optical waveguides with astigmatically shaped beams. J Opt Soc Am B 2003; 20: 1559-1567.

97 Sugioka K, Cheng Y, Midorikawa K, Takase F, Takai H. Femtosecond laser microprocessing with three-dimensionally isotropic spatial resolution using crossedbeam irradiation. Opt Lett 2006; 31: 208-210.

$98 \mathrm{He} \mathrm{F}$, Cheng Y, Lin JT, Ni JL, Xu ZZ et al. Independent control of aspect ratios in the axial and lateral cross sections of a focal spot for three-dimensional femtosecond laser micromachining. New J Phys 2011; 13: 083014.

99 Thomson RR, Kar AK, Allington-Smith J. Ultrafast laser inscription: an enabling technology for astrophotonics. Opt Express 2009; 17: 1963-1969.

100 Marshall GD, Politi A, Matthews JC, Dekker P, Ams M et al. Laser written waveguide photonic quantum circuits. Opt Express 2009; 17: 12546-12554.

101 Crespi A, Osellame R, Ramponi R, Brod DJ, Galvão EF et al. Integrated multimode interferometers with arbitrary designs for photonic boson sampling. Nat Photonics 2013; 7: 545-549.

102 Marcinkevičius A, Juodkazis S, Watanabe M, Miwa M, Matsuo S et al. Femtosecond laser-assisted three-dimensional microfabrication in silica. Opt Lett 2001; 26: 277 279. 
103 Masuda M, Sugioka K, Cheng Y, Aoki N, Kawachi M et al. 3-D microstructuring inside photosensitive glass by femtosecond laser excitation. App/ Phys A 2003; 76: 857860.

104 Li Y, Itoh K, Watanabe W, Yamada K, Kuroda D et al. Three-dimensional hole drilling of silica glass from the rear surface with femtosecond laser pulses. Opt Lett 2001; 26: 1912-1914.

105 Liao Y, Ju YF, Zhang L, He F, Zhang Q et al. Three-dimensional microfluidic channel with arbitrary length and configuration fabricated inside glass by femtosecond laser direct writing. Opt Lett 2010; 35: 3225-3227.

106 Liao Y, Song J, Li E, Luo Y, Shen YL et al. Rapid prototyping of three-dimensional microfluidic mixers in glass by femtosecond laser direct writing. Lab Chip 2012; 12: 746-749.

107 Liao Y, Shen YL, Qiao LL, Chen D, Cheng Y et al. Femtosecond laser nanostructuring in porous glass with sub-50 nm feature sizes. Opt Lett 2013; 38: 187-189.

108 Cheng Y, Sugioka K, Midorikawa K. Microfluidic laser embedded in glass by threedimensional femtosecond laser microprocessing. Opt Lett 2004; 29: 2007-2009.

109 Hanada Y, Sugioka K, Kawano H, Ishikawa IS, Miyawaki A et al. Nano-aquarium for dynamic observation of living cells fabricated by femtosecond laser direct writing of photostructurable glass. Biomed Microdevices 2008; 10: 403-410.

110 Hanada Y, Sugioka K, Shihira-Ishikawa I, Kawano H, Miyawaki A et al. 3D microfluidic chips with integrated functional microelements fabricated by a femtosecond laser for studying the gliding mechanism of cyanobacteria. Lab Chip 2011; 11: 2109-2115.

111 Crespi A, Gu Y, Ngamsom B, Hoekstra HJ, Dongre C et al. Three-dimensional MachZehnder interferometer in a microfluidic chip for spatially-resolved label-free detection. Lab Chip 2010; 10: 1167-1173.

112 Hanada Y, Sugioka K, Midorikawa K. Highly sensitive optofluidic chips for biochemical liquid assay fabricated by $3 D$ femtosecond laser micromachining followed by polymer coating. Lab Chip 2012; 12: 3688-3693.

$113 \mathrm{Kim}$ M, Hwang DJ, Hiromatsu K, Grigoropoulos CP. Single cell detection using a glassbased optofluidic device fabricated by femtosecond laser pulses. Lab Chip 2009; 9: 311-318.

114 Bragheri F, Ferrara L, Bellini N, Vishnubhatla KC, Minzioni P et al. Optofluidic chip for single cell trapping and stretching fabricated by a femtosecond laser. J Biophotonics 2010; 3: 234-243.

115 Choudhury D, Ramsay WT, Kiss R, Willoughby NA, Paterson L et al. A 3D mammalian cell separator biochip. Lab Chip 2012; 12: 948-953.

116 Schaap A, Rohrlack T, Bellouard Y, Lab on a chip technologies for algae detection: a review. J Biophotonics 2012; 5: 661-672.

117 Cheng Y, Sugioka K, Midorikawa K, Masuda M, Toyoda K et al. Three-dimensional micro-optical components embedded in photosensitive glass by a femtosecond laser. Opt Lett 2003; 28: 1144-1146.

118 Wang Z, Sugioka K, Midorikawa K. Three dimensional integration of microoptical components buried inside photosensitive glass by femtosecond laser direct writing. Appl Phys A 2007; 89: 951-955.

119 He F, Cheng Y, Qiao LL, Wang C, Xu ZZ et al. Two-photon fluorescence excitation with a microlens fabricated on the fused silica chip by femtosecond laser micromachining. Appl Phys Lett 2010; 96: 041108.

120 Shimotsuma Y, Sakakura M, Kazansky PG, Beresna M, Qiu JR et al. Ultrafast manipulation of self-assembled form birefringence in glass. Adv Mater 2010; 22: 4039-4043.
121 Beresna M, Gecevičius M, Kazansky PG. Polarization sensitive elements fabricated by femtosecond laser nanostructuring of glass. Opt Mater Express 2011; 1: 783-795.

122 Sugioka K, Gu B, Holmes A. The state of the art and future prospects for laser directwrite for industrial and commercial applications. MRS Bull 2007; 32: 47-54.

123 Haight R, Wagner A, Longo P, Lim D. Femtosecond laser ablation and deposition of metal films on transparent substrates with applications in photomask repair. Proc SPIE 2005; 5714: 24-36.

124 Abeln T, Klink U. Laseroberflächenstrukturierung-Verbesserung der tribologischen Eigenschaften. In: Proceedings of Stuttgarter Lasertage 2003; 25-26 September 2003; Stuttgart, Germany. FGSW: Stuttgart, Germany, 2003; 107-110.

125 Bauer Th, König J. Applications and perspectives of ultrashort pulsed lasers. Tech Dig LPM 2010; 2010: 127.

126 SiOnyx solar achieves record results for black silicon solar cells: http://sionyx.com/ 2011/10/sionyx-solar-achieves-record-results-for-black-silicon-solar-cells-2/.

127 Booth H. Laser processing in industrial solar module manufacturing. J Laser Micro/ Nanoeng 2010; 5: 183-191.

128 Weiler S. High-power pico- and femtosecond lasers enable new applications. Laser Focus World 2011; 47: 55-63.

129 Tönshoff HK, Ostendorf A, Nolte S, Korte F, Bauer T. Micromachining using femtosecond lasers. Proc SPIE 2000; 4088: 136-139.

130 Binder PS. Flap dimensions created with the IntraLase FS laser. J Cataract Refract Surg 2004; 30: 26-32.

131 IntraLase ${ }^{\mathrm{TM}}$ FS Laser: http://www.amo-inc.com/products/refractive/ilasik/intralase-fslaser.

132 Gottmann J, Hermans M, Ortmann J. Microcutting and hollow 3D microstructures in glasses by in-volume selective laser-induced etching (ISLE). J Laser Micro/Nanoeng 2013; 8: 15-18.

133 Tünnermann A, Schreiber T, Limpert J. Fiber lasers and amplifiers: an ultrafast performance evolution. App/ Opt 2010; 49: F71-F78.

$134 \mathrm{He} \mathrm{F}, \mathrm{Xu} \mathrm{H}$, Cheng Y, Ni JL, Xiong H et al. Fabrication of microfluidic channels with a circular cross section using spatiotemporally focused femtosecond laser pulses. Opt Lett 2010; 35: 1106-1108.

135 Vitek DN, Adams DE, Johnson A, Tsai PS, Backus S et al. Temporally focused femtosecond laser pulses for low numerical aperture micromachining through optically transparent materials. Opt Express 2010; 18: 18086-18094.

136 Zeng B, Chu W, Gao H, Liu WW, Li GH et al. Enhancement of peak intensity in a filament core with spatiotemporally focused femtosecond laser pulses. Phys $\operatorname{Rev} A$ 2011; 84: 063819.

137 Kim D, So PT. High-throughput three-dimensional lithographic microfabrication. Opt Lett 2010; 35: 1602-1604.

138 Vitek DN, Block E, Bellouard Y, Adams DE, Backus S et al. Spatio-temporally focused femtosecond laser pulses for nonreciprocal writing in optically transparent materials. Opt Express 2010; 18: 24673-24678.

139 Bellouard Y, Champion A, Lenssen B, Matteucci M, Schaap A et al. The femtoprint project. J Laser Micro/Nanoeng 2012; 7: 1-10.

(c) (i) (3) $\Theta$ This work is licensed under a Creative Commons AttributionNo No Nommercial-NoDerivs Works 3.0 Unported license. To view a copy of this license, visit http://creativecommons.org/licenses/by-nc-nd/3.0 